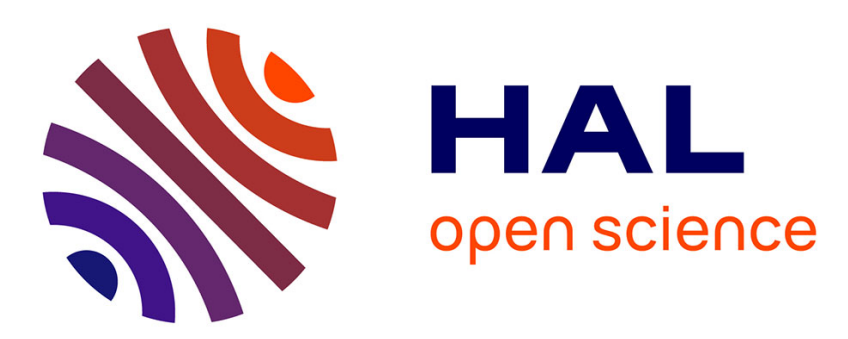

\title{
On models of layered piezoelectric beams for passive vibration control
}

Corrado Maurini, Francesco Dell'Isola, Joël Pouget

\section{To cite this version:}

Corrado Maurini, Francesco Dell'Isola, Joël Pouget. On models of layered piezoelectric beams for passive vibration control. Journal de Physique IV Proceedings, 2004, pp.10. hal-00497029

\section{HAL Id: hal-00497029 \\ https://hal.science/hal-00497029}

Submitted on 2 Jul 2010

HAL is a multi-disciplinary open access archive for the deposit and dissemination of scientific research documents, whether they are published or not. The documents may come from teaching and research institutions in France or abroad, or from public or private research centers.
L'archive ouverte pluridisciplinaire HAL, est destinée au dépôt et à la diffusion de documents scientifiques de niveau recherche, publiés ou non, émanant des établissements d'enseignement et de recherche français ou étrangers, des laboratoires publics ou privés. 


\title{
On models of layered piezoelectric beams for passive vibration control
}

\author{
C. Maurini ${ }^{1,2}$, F. dell'Isola ${ }^{3}$ and J. Pouget ${ }^{1}$ \\ ${ }^{1}$ LEMA (CNRS-FRE 2481), Université de Versailles/Saint-Quentin-en-Yvelines, $45 \mathrm{Av}$. \\ des États-Unis, 78035 Versailles, France \\ e-mail: joel.pouget@meca.uvsq.fr \\ ${ }^{2}$ Dipartimento di Meccanica e Aeronautica, Univertità di Roma "La Sapienza", via Eudossiana \\ 18, 00184 Roma, Italy \\ ${ }^{3}$ Dipartimento di Ingegneria Strutturale \& Geotecnica, Univertità di Roma "La Sapienza", via \\ Eudossiana 18, 00184 Roma, Italy
}

\begin{abstract}
In this paper models of layered piezoelectric beams are discussed. The attention is focused on the analysis of the assumptions on transversal stress and strain distribution and their influence on the deduction of the beam constitutive equations from a three dimensional description. A model accounting for non trivial transversal interactions between different layers is deduced from a mixed variational formulation where non-local conditions on transversal stress are enforced by Lagrange multipliers method. The fully coupled electromechanical nature of the system is described. For a sandwich piezoelectric beam, analytical expressions of the beam constitutive coefficients are provided and comparisons to standard modelling approaches are presented. Finally, the fundamental features of the proposed model are highlighted by presenting the through-the-thickness distribution of the 3D state fields.
\end{abstract}

\section{INTRODUCTION}

Composite materials made of active piezoelectric layers have been widely used for their sensor and actuator functions and research on this area opens many applications in the domain of adaptive structures and structural control. Piezoelectric materials, and especially piezoelectric beam composites, are excellent candidates for designing adaptive smart structures, the mechanical properties of which are controlled through, either active or passive, electronic devices [1]. A proper design of the electronic control systems requires, as a preliminary step, an efficient modelling of the electromechanical properties of the mechanical system, the transduction devices and of the interactions between them.

The present work attempts to present a consistent and efficient one-dimensional model of beamshaped, laminated piezoelectric composites taking into account a two-fold electromechanical coupling and two-dimensional interactions between elastic and piezoelectric layers. Although a number of accurate beam models have been presented (see [2] for a review), most of them do not describe the fully coupled electro-mechanical nature of the system (the piezoelectric elements are regarded merely as either actuators [3] or sensors [4]) and neglect transversal interactions between elastic and piezoelectric layers by assuming either pointwise vanishing transversal stress or strain (this issue is discussed in [5]). As a consequence, global properties as the equivalent piezoelectric capacitance are not correctly estimated. In the following, by carefully choosing the hypotheses on 3D state field distribution, we aim to develop a simple beam model based on an Euler-Bernoulli kinematics in which the electromechanical 
constitutive coefficients are estimated with an acceptable approximation. By means of mixed variational formulation [6], hypotheses on both kinematical and dynamic state fields are rationally included in the model. Non trivial transversal interactions between different layers are taken into account by replacing standard local conditions on transversal field distributions by integral, weakened versions which are enforced in the variational formulation through Lagrange multipliers method.

\section{BEAM MODEL}

Consider a multilayered straight-axis piezoelectric beam which is composed by stacking up $n$ rectangular cross-section piezoelectric and elastic layers with width $a_{i}$ and thickness $h_{i}$. We denote by $\mathcal{A}$ the beam axis, by $\mathcal{S}$ the beam cross-section, and by $\mathcal{S}_{i}$ the cross sectional part occupied by the $i$-th layer. The following geometric and material properties are assumed: i) each layer is materially homogeneous and either orthotropic or transversely isotropic with respect to an axis oriented along its thickness (in particular the piezoelectric layers are polarized along the thickness); ii) the upper and lower surfaces of the piezoelectric layers are covered by a conductive layer with negligible mechanical properties, the lateral ones are bared; iv) the electrodes of the piezoelectric layers are connected in parallel one to each other, and the whole beam is electrically accessible only through two external electric terminals. For each piezoelectric layer, we define a constant $\omega_{i}= \pm 1$ which determines the electric connection scheme between the electrodes of the $i-t h$ layer and the external terminals $\left(\omega_{i}=1\right.$ in-phase connection, $\omega_{i}=-1$ counter-phase connection). A global Cartesian reference frame $\mathcal{C}=\left\{o, \mathbf{e}_{1}, \mathbf{e}_{2}, \mathbf{e}_{3}\right\}$, oriented in such a way that $\mathbf{e}_{1}$ is aligned along the beam axis, $\mathbf{e}_{2}$ along the cross sectional width and $\mathbf{e}_{3}$ along the cross sectional thickness, is fixed once for all. Moreover $n$ local reference frames $\mathcal{C}_{i}=\left\{o_{i}, \mathbf{e}_{1}, \mathbf{e}_{2}, \mathbf{e}_{3}\right\}$, with $o_{i}=o+\bar{z}_{i} \mathbf{e}_{3}$, are introduced. We assume that each $\bar{z}_{i}$ is chosen such that:

$$
\int_{S_{i}} z_{i} d S=0 .
$$

For each layer we define the following sectional area and moment of inertia

$$
A_{i}=\int_{S_{i}} d S, \quad J_{i}=\int_{S_{i}} z_{i}^{2} d S .
$$

The beam axis plane motion in the $\mathbf{e}_{1}-\mathbf{e}_{3}$ plane is analyzed and the following external actions are accounted for: i) a surface force distribution $\mathbf{f}(x, y, z)$ on the beam bases having a force resultant $\mathbf{F}=\bar{N} \mathbf{e}_{1}+\bar{T} \mathbf{e}_{3}$ and a moment resultant $\mathbf{M}=-\bar{M} \mathbf{e}_{2} ;$ ii) a body force per unit of volume $\mathbf{b}(x, y, z)$, having over a section a force and moment resultants per unit of line $b_{N}(x) \mathbf{e}_{1}+b_{T}(x) \mathbf{e}_{3}$ and $-b_{M}(x) \mathbf{e}_{2}$, respectively; iii) either a voltage $\bar{V}$ or a total charge $\bar{Q}$ imposed on the electric terminal of the set of piezoelectric layers.

\subsection{Hypotheses}

Under the quasi-electrostatic approximation, the kinematic state of a three-dimensional piezoelectric Continuum can be determined by the vector field $\mathbf{u}$ and a scalar field $\varphi$, representing the mechanical displacement and the electric potential with respect to a reference configuration $\mathcal{B}$. The corresponding generalized deformations are the mechanical strain tensor $\mathbf{S}=\operatorname{Sym}(\nabla \mathbf{u})$ and the electric field vector $\mathbf{E}=-\nabla \varphi$. The associated generalized forces are the Cauchy stress tensor $\mathbf{T}$ and the electric displacement vector $\mathbf{D}$. By adopting a direct approach, reduced models of a given structure can be deduced from the three dimensional description by assuming specific distribution of the state fields. In this paper, a beam model based on the following hypotheses on the electromechanical state-fields is presented.

(H1) Mechanical displacement: basic equivalent-single-layer Euler-Bernoulli kinematics

$$
\mathbf{u}(x, y, z)=\left(u(x)-z w^{\prime}(x)\right) \mathbf{e}_{1}+w(x) \mathbf{e}_{3}
$$

where $u(x)$ and $w(x)$ are the beam axis displacements along $\mathbf{e}_{1}$ and $\mathbf{e}_{3}$, respectively. 
(H2) Electric potential: layerwise quadratic distribution of the electric potential and parallel interconnection between the different layers

$$
\varphi(x, y, z)=\left(\frac{1}{2}+\omega_{i} \frac{z_{i}}{h_{i}}\right) V+\frac{1}{2}\left(\frac{h_{i}^{2}}{4}-z_{i}^{2}\right) \phi_{i}(x),
$$

where $V$ is the electric potential difference applied between the two external electric terminals and $\phi_{i}(x)$ describes the intensity of the quadratic contribution to the electric potential in the $i-t h$ layer.

(H3) Mechanical stress: layerwise linear distribution of plane-stress with vanishing shear term

$$
\mathbf{T}(x, y, z)=\left(\sigma_{i}^{(1)}(x)-z_{i} \zeta_{i}^{(1)}(x)\right)\left(\mathbf{e}_{1} \otimes \mathbf{e}_{1}\right)+\left(\sigma_{i}^{(2)}(x)-z_{i} \zeta_{i}^{(2)}(x)\right)\left(\mathbf{e}_{2} \otimes \mathbf{e}_{2}\right),
$$

where, layer by layer, constant $\left(\sigma^{\prime} s\right)$ and linear $\left(\zeta^{\prime} s\right)$ contributions to the beam stress in the axial $\left(\mathbf{e}_{1}\right)$ and transversal $\left(\mathbf{e}_{2}\right)$ directions are defined.

(H4) Electric displacement: layerwise constant distribution along the thickness direction

$$
\mathbf{D}(x, y, z)=\mathrm{D}_{i}(x) \mathbf{e}_{3}
$$

The important assumption in hypotheses (5) consists of prescribing that the normal stress in the $\mathbf{e}_{3}$ direction is vanishing. Indeed, with this hypotheses a sectional distension in the $\mathbf{e}_{3}$ direction is implicitly accounted for at constitutive level (see [7] for a discussion of the analog problem in elastic plates). Also hypotheses (4) and (6) worth a comment: by assuming a constant distribution of the electric displacement along the thickness, the quadratic part of the electric potential has no energy contributions and, although not vanishing, it do not influence the distributions of other beam state fields. The hypothesis of constant electric displacement replaces the one, frequently assumed, of linear electric potential. The latter implies systematic errors in the evaluation of the beam constitutive coefficients.

In a beam model accounting for hypotheses (3-6), the beam constitutive equations are derived from the three dimensional ones by assuming that, when the beam axis deforms, the cross sections remain rigid along the transversal direction $\mathbf{e}_{2}$ (hypthesis 3 ). In order to enforce this constraint, transversal reactive stresses arise and $\left(\sigma_{i}^{(2)}, \zeta_{i}^{(2)}\right)$ are in general different from zero. When the beam mantle is stress free and when no body forces are applied on the transversal direction, this condition is not realistic and the beam should be left free to stretch along $\mathbf{e}_{2}$. For this reason, in standard beam model the following condition of pointwise vanishing transversal stress is usually enforced at constitutive level

$$
\mathbf{T}(x, y, z) \cdot\left(\mathbf{e}_{2} \otimes \mathbf{e}_{2}\right)=0 .
$$

This assumption is usually motivated by the stress free conditions on the beam lateral boundary. However, for layered beams, one should consider that interactions between different layers arise and, due to the prescribed single layer kinematics (3), the 3D equilibrium equations and boundary conditions have not to be satisfied pointwise (refer to [8] for a discussion of the role of constraints and reactive stresses in beams). Because of this incongruence, layered beam models based on hypothesis (7) fail to predict some relevant phenomena as the interaction between different layers in the transversal direction. In the following sections, we show that these phenomena can be described by a beam model where condition (7) is replaced by the following weakened versions (vanishing stress resultants)

$$
\left\{\begin{array}{l}
N_{y}=\int_{\mathcal{S}} \mathbf{T}(x, y, z) \cdot\left(\mathbf{e}_{2} \otimes \mathbf{e}_{2}\right) d \mathcal{S}=\sum_{i \in \mathcal{I}} A_{i} \sigma_{i}^{(2)}=0 \\
M_{y}=\int_{\mathcal{S}}-z \mathbf{T}(x, y, z) \cdot\left(\mathbf{e}_{2} \otimes \mathbf{e}_{2}\right) d \mathcal{S}=\sum_{i \in \mathcal{I}} J_{i} \zeta_{i}^{(2)}-\bar{z}_{i} A_{i} \sigma_{i}^{(2)}=0
\end{array}\right.
$$


which are physically justified by global balance laws over a section and, differently from (7), holds also when the kinematical constraint (3) is prescribed. Here and henceforth, we refer to (7) and (8) as strong condition (SC) and weak condition (WC) on transversal stress, respectively. In the following, a beam model based on hypotheses (3-6) and the WC (8) is derived. The corresponding constitutive equations are compared to those obtained by assuming either vanishing transversal strain (condition 36) or pointwise vanishing transverse stress (conditions (3-6) and SC (7)).

\subsection{Mixed variational formulation}

A beam model accounting for hypotheses (3-6) can be derived from the description at three dimensional level by using a mixed variational formulation based on the Hellinger-Prange-Reissner functional for piezoelectricity. The additional conditions (8) on transversal stress are included in the variational formulation by means of the Lagrange multipliers method.

Let us consider a three dimensional piezoelectric continuum $\mathcal{B}$ on which the following external actions are applied: body forces $\mathbf{b}$ on the bulk $\mathcal{B}$, surface forces $\mathbf{f}_{0}$ and surface charges $q_{0}$ on the parts $\partial_{f} \mathcal{B}$ and $\partial_{q} \mathcal{B}$ of the boundary $\partial \mathcal{B}$, mechanical displacement $\mathbf{u}_{0}$ and electric potential $\varphi_{0}$ on the parts $\partial_{\mathbf{u}} \mathcal{B}$ and $\partial_{\varphi} \mathcal{B}$ of $\partial B$. Let be $\mathcal{V}_{\mathbf{u}}$ and $\mathcal{V}_{\varphi}$ the functional spaces of kinematically compatible ${ }^{\mathrm{a}}$ mechanical displacement and strain pairs $(\mathbf{u}, \mathbf{S})$ and electric potential and electric field pairs $(\varphi, \mathbf{E})$. Moreover let be $\mathcal{V}_{\mathbf{T}}$ and $\mathcal{V}_{\mathbf{D}}$ the spaces of admissible stress tensors $\mathbf{T}$, and electric displacement vectors $\mathbf{D}$. The solution of the problem of three dimensional piezoelectricity is characterized by rendering stationary the following functional (Hellinger-Prange-Reissner functional of piezoelectricity) over the space $\mathcal{V}=$ $\mathcal{V}_{\mathbf{u}} \times \mathcal{V}_{\varphi} \times \mathcal{V}_{\mathbf{T}} \times \mathcal{V}_{\mathbf{D}}$

$$
\Theta[(\mathbf{u}, \mathbf{S}),(\varphi, \mathbf{E}), \mathbf{T}, \mathbf{D}]=\int_{\mathcal{B}}(\mathcal{F}(\mathrm{T}, \mathrm{D})-\mathbf{T} \cdot \mathbf{S}-\mathbf{D} \cdot \mathbf{E}+\mathbf{b} \cdot \mathbf{u}) d \mathcal{B}+\int_{\partial_{\mathrm{f}} \mathcal{B}} \mathbf{f}_{0} \cdot \mathbf{u} d \mathcal{S}-\int_{\partial_{\mathrm{q}} \mathcal{B}} q_{0} \varphi d \mathcal{S}
$$

The scalar valued function $\mathcal{F}(\mathbf{T}, \mathbf{D})$ is a piezoelectric internal energy density defined by

$$
\mathcal{F}(\mathbf{T}, \mathbf{D})=\frac{1}{2} s^{D} \mathbf{T} \cdot \mathbf{T}-\frac{1}{2} \beta^{T} \mathbf{D} \cdot \mathbf{D}+\mathrm{g} \mathbf{T} \cdot \mathbf{D}
$$

where $\mathrm{s}^{D}$ is the forth order elastic compliance tensor for null electric displacement, $\beta^{T}$ the second order dielectric constants tensor for null mechanical stress, $g$ the third order piezoelectric coupling tensor.

The equations of 3D linear piezoelectricity are equivalent to the Euler equations of the functional (9): the mechanical and electrical balance equations and natural boundary conditions correspond to the Euler-equations in $\mathcal{V}_{\mathbf{u}}$ and $\mathcal{V}_{\varphi}$, the constitutive equations by those in $\mathcal{V}_{\mathbf{T}}$ and $\mathcal{V}_{\mathbf{D}}$. The essential boundary conditions on $\mathbf{u}$ and $\varphi$ and the kynematical compatibility equations are included in the definitions of the functional spaces $\mathcal{V}_{\mathbf{u}}$ and $\mathcal{V}_{\varphi}$.

In a beam model accounting for hypotheses (3-6), the distributions of the three dimensional state fields $(\mathbf{u}, \varphi, \mathbf{T}, \mathbf{D})$ is uniquely determined by the axial fields $\left(u, w, \phi_{i}, \sigma_{i}^{(\alpha)}, \zeta_{i}^{(\alpha)}, D_{i}\right)$ and the voltage difference $V$ (beam state fields). Here and henceforth the mute indices $\alpha$ and $i$ are intended to vary from 1 to 2 and from 1 to $n$, respectively. By introducing hypotheses (3-6) into the functional (9) it is possible to deduce the beam functional

$$
\Theta_{\text {beam }}\left[u, w, \sigma_{i}^{(\alpha)}, \zeta_{i}^{(\alpha)}, D_{i}, V\right]
$$

which is defined over $\mathcal{W}$, functional space of admissible beam state fields. The variational formulation corresponding to a beam model accounting for hypotheses (3-6) and the weak conditions (8) on

${ }^{\mathrm{a}} \mathrm{S}=\operatorname{sym}(\nabla \mathbf{u})$ on $\mathcal{B}$ and $\mathbf{u}=\mathbf{u}_{0}$ on $\partial_{\mathbf{u}} \mathcal{B} ; \mathbf{E}=-\nabla \varphi$ on $\mathcal{B}$ and $\varphi=\varphi_{0}$ on $\partial_{\varphi} \mathcal{B}$ 
transversal stresses can be obtained by Lagrange multipliers methods through the definition of the modified functional

$$
\Theta_{\text {beam }}^{\Lambda}\left[u, w, \sigma_{i}^{(\alpha)}, \zeta_{i}^{(\alpha)}, D_{i}, V, \lambda, \mu\right]=\Theta_{\text {beam }}\left[u, w, \sigma_{i}^{(\alpha)}, \zeta_{i}^{(\alpha)}, D_{i}, V\right]-\int_{\mathcal{A}} \lambda N_{y} d x-\int_{\mathcal{A}} \mu M_{y} d x
$$

where $\lambda$ and $\mu$ are Lagrange multipliers axial fields enforcing, section by section, the conditions (8) of vanishing transversal force and moment resultant. The corresponding variational formulation consists of looking for those beam state fields in $\mathcal{W}$ and those admissible Lagrange multipliers $(\lambda, \mu)$ rendering stationary the functional (12). The Lagrange multipliers $\lambda$ and $\mu$ are the transversal analogs of the axial deformations $u^{\prime}$ and $w^{\prime \prime}$ : they can be interpreted as constant $(\lambda)$ and linear $(\mu)$ parts of the transversal deformation of the beam cross section.

In a model derived by a mixed variational formulation, it is necessary to distinguish between the generalized deformations $(\mathbf{S}, \mathbf{E})$ related to the generalized displacements $(\mathbf{u}, \varphi)$ through the kinematic compatibility and the generalized deformations $(\overline{\mathbf{S}}, \overline{\mathbf{E}})$ related to generalized stresses $(\mathbf{T}, \mathbf{D})$ though the constitutive equations. When reduced models are considered, $(\mathbf{S}, \mathbf{E})$ are deduced by hypotheses on generalized displacements, $(\overline{\mathbf{S}}, \overline{\mathbf{E}})$ by hypotheses on generalized stresses and, in general, they do not coincide. The former are utilized in the deduction of the beam balance equations, the latter are those taken into account when beam constitutive equations are derived by the 3D ones. In the present model, while $(\mathbf{S}, \mathbf{E})$ are the deformations corresponding to standard Euler-Bernoulli kinematics with rigid cross section, $(\overline{\mathbf{S}}, \overline{\mathbf{E}})$ include linear transversal sectional deformations and layerwise linear sectional distensions along the thickness (see Fig. 2).

\subsection{Beam governing equations}

By imposing that the functional (12) is stationary for all the admissible beam state fields and for all the admissible Lagrange multiplier fields $(\lambda, \mu)$, we obtain: i) beam balance equations and natural boundary conditions (from variations with respect to $u, w$, and $V$ ); ii) beam constitutive equations (from variations with respect to $\sigma_{i}^{(\alpha)}, \zeta_{i}^{(\alpha)}$, and $D_{i}$ ); iii) the conditions (8) on transversal stresses (from variations with respect to $\lambda$ and $\mu$ ).

The beam balance equations and boundary conditions are

$$
\begin{aligned}
& b_{N}+N_{x}^{\prime}=0, b_{M}^{\prime}-b_{T}+M_{x}^{\prime \prime}=0, \int_{\mathcal{A}} q d x+\bar{Q}=0, \\
& {\left[\left(M_{x}-\bar{M}\right) \delta w^{\prime}+\left(\bar{T}+M_{x}^{\prime}\right) \delta w\right]_{\partial \mathcal{A}}=0,\left[\left(N_{x}-\bar{N}\right) \delta u\right]_{\partial \mathcal{A}}=0,}
\end{aligned}
$$

where

$$
N_{x}=\sum_{i \in \mathcal{I}} A_{i} \sigma_{i}^{(1)}, \quad M_{x}=\sum_{i \in \mathcal{I}} J_{i} \zeta_{i}^{(1)}-\sum_{i \in \mathcal{I}} \bar{z}_{i} A_{i} \sigma_{i}^{(1)}, q=-\sum_{i \in \mathcal{I}_{p}} \omega_{i} \mathrm{D}_{i} A_{i} / h_{i}
$$

are resultant normal stress, bending moment and charge per unit of line. Because of the assumed kinematics, the mechanical equilibrium equations are those of a standard Euler-Bernoulli beam, the electric one is the Kirchhoff law at the external electric terminals. If the constitutive equations for the beam state fields are written in a suitable from, they can be directly substituted in the definitions (14). Hence, the following constitutive equations explicitly including transversal deformations and stress resultants are obtained

$$
\left[\begin{array}{c}
\mathrm{R}_{x} \\
\mathrm{R}_{y} \\
q
\end{array}\right]=\left[\begin{array}{ccc}
K_{x x}^{0} & K_{x y}^{0} & \left(e_{d V}^{0}\right)^{t} \\
K_{x y}^{0} & K_{x x}^{0} & \left(e_{d V}^{0}\right)^{t} \\
-e_{d V}^{0} & -e_{d V}^{0} & \epsilon_{q V}^{0}
\end{array}\right]\left[\begin{array}{c}
\mathrm{d}_{x} \\
\mathrm{~d}_{y} \\
V
\end{array}\right]
$$

where $\mathrm{R}_{x}=\left\{N_{x}, M_{x}\right\}^{t}, \mathrm{R}_{y}=\left\{N_{y}, M_{y}\right\}^{t}, \mathrm{~d}_{x}=\left\{u^{\prime}, w^{\prime \prime}\right\}^{t}, \mathrm{~d}_{y}=\{\lambda, \mu\}^{t}$, and

$$
K_{x x}^{0}=\left[\begin{array}{cc}
K_{N u}^{0} & -K_{N w}^{0} \\
-K_{N w}^{0} & K_{M w}^{0}
\end{array}\right], K_{x y}^{0}=\left[\begin{array}{cc}
K_{N \lambda}^{0} & -K_{N \mu}^{0} \\
-K_{N \mu}^{0} & K_{M \mu}^{0}
\end{array}\right], e_{d V}^{0}=\left[\begin{array}{ll}
-e_{N V}^{0} & e_{M V}^{0}
\end{array}\right],
$$


Table 1. Beam model constitutive coefficients

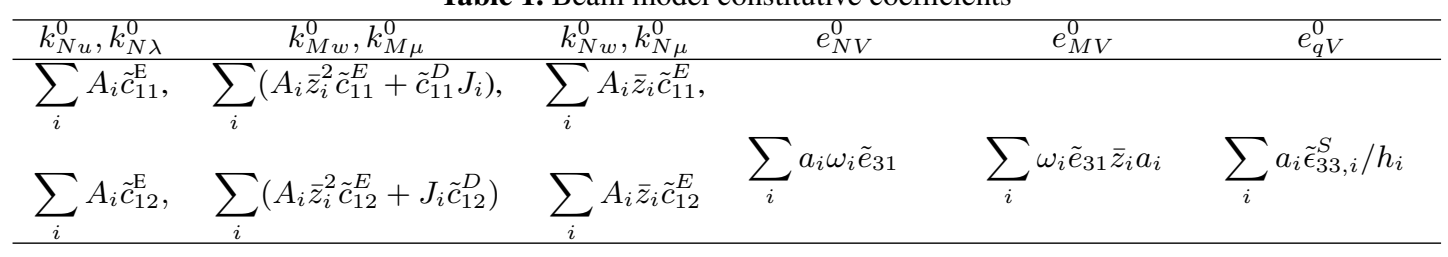

The constitutive coefficients above are functions of the material and geometrical properties of the three dimensional model. Their explicit expressions are given in Table 1, where standard notation for piezoelectric constitutive coefficients is adopted [9]. The coefficients with a superscripted $\sim$ refer to plane stress condition (in the $\mathrm{e}_{1}-\mathrm{e}_{2}$ plane), those with a superscripted $\hat{\imath}$ to uniaxial stress condition (along $\mathrm{e}_{1}$ ). They can be conveniently evaluated from the constitutive properties given in Table 2 through the following, relations ( $s_{11}^{E}$ and $s_{12}^{E}$ are elastic compliances for null electric field, $\varepsilon_{33}^{T}$ is a dielectric constant for null mechanical stress, and $d_{31}$ is an electromechanical coupling coefficient)

$$
\begin{array}{rlrl}
\tilde{c}_{11}^{E} & =\frac{s_{11}^{E}}{\left(s_{11}^{E}\right)^{2}-\left(s_{12}^{E}\right)^{2}}, & \tilde{c}_{12}^{E}=\frac{s_{12}^{E}}{\left(s_{11}^{E}\right)^{2}-\left(s_{12}^{E}\right)^{2}}, & \tilde{e}_{31}=\frac{d_{31}}{s_{11}^{E}+s_{12}^{E}}, \\
\hat{c}_{11}^{E}=\frac{1}{s_{11}^{E}}, & \hat{\varepsilon}_{31}^{T}=\frac{d_{31}}{s_{11}^{E}}, & \hat{\varepsilon}_{33}^{T}=\varepsilon_{33}^{T}-\frac{d_{31}^{T}}{s_{11}^{E}}, \hat{c}_{11}^{D}=\hat{c}_{11}^{E}+\hat{e}_{31}^{2} / \hat{\varepsilon}_{33}^{T}
\end{array}
$$

Moreover, the mechanical stiffnesses for null electric displacements $\left(\tilde{c}_{11}^{D}, \tilde{c}_{12}^{D}\right)$ are given by $\left(\tilde{c}_{11}^{D}\right)=$ $\tilde{c}_{11}^{E}+\tilde{e}_{31}^{2} / \tilde{\varepsilon}_{33}^{T}$ and $\tilde{c}_{12}^{D}=\tilde{c}_{12}^{E}+\tilde{e}_{31}^{2} / \tilde{\varepsilon}_{33}^{T}$. For the elastic layers, one can simply assume $c_{h k}^{E}=c_{h k}^{D}=c_{h k}$, $\tilde{c}_{h k}^{E}=\tilde{c}_{h k}^{D}=\tilde{\tilde{c}}_{h k}, e_{m k}=\tilde{e}_{m k}=0, \varepsilon_{m l}^{T}=\tilde{\varepsilon}_{m l}^{T}=0$. The matrices $K_{x x}^{0}, e_{d V}^{0}, \varepsilon_{q V}^{0}$ are the beam constitutive coefficients corresponding to a beam model assuming null transversal strain $\left(\mathrm{d}_{y}=0\right)$. In the present case (weak conditions on transversal stress), the transversal sectional deformations $\mathrm{d}_{y}$ are different from zero. The corresponding expressions as a function of the axial deformations and the applied voltage are found by imposing the conditions $\mathrm{R}_{y}=0$ (i.e. vanishing variations of the functional (12) with respect to the Lagrange multipliers)

$$
\mathrm{d}_{y}=-\left(K_{x x}^{0}\right)^{-1} K_{x y}^{0} \mathrm{~d}_{x}-\left(K_{x x}^{0}\right)^{-1}\left(e_{d V}^{0}\right)^{t} V
$$

Hence, by substituting the expression (18) in (15), the following beam model constitutive equations are found

$$
\left[\begin{array}{c}
\mathrm{R}_{x} \\
q
\end{array}\right]=\left[\begin{array}{cc}
K_{x x} & e_{d V}^{t} \\
-e_{d V} & \epsilon_{q V}
\end{array}\right]=\left[\begin{array}{c}
\mathrm{d}_{x} \\
V
\end{array}\right]
$$

where the novel beam constitutive matrices implicitly take into account the weak conditions (8) on transversal stresses and are given by

$$
K_{x x}=K_{x x}^{0}-K_{x y}^{0}\left(K_{x x}^{0}\right)^{-1} K_{x y}^{0}, e_{d V}=e_{d V}^{0}-e_{d V}^{0}\left(K_{x x}^{0}\right)^{-1} K_{x y}^{0}, \epsilon_{q V}=\epsilon_{q V}^{0}+\epsilon_{d V}^{0}\left(K_{x x}^{0}\right)^{-1}\left(e_{d V}^{0}\right)^{t} .
$$

\section{RESULTS AND COMPARISONS FOR A SANDWICH BEAM}

If essential boundary conditions are prescribed on $u$ and $w$, the beam balance equations and natural boundary conditions(13) and the constitutive equations (19) lead to the complete set of equations for the beam model assuming hypotheses (3-6) and the weak conditions on transversal stresses (8). When the kinematical hypotheses (3-4) are assumed, different conditions on the distribution of the generalized 
stresses (T, D) lead to different constitutive coefficients for the beam constitutive equations (19). The model presented above assumes vanishing transversal stress resultants (weak conditions (8)). In order to analyze the consequences of different assumptions on transversal stress distribution, this model is compared to the ones assuming either pointwise vanishing transversal stress (strong condition (7)) or pointwise vanishing transversal strain (no further conditions in addition to hypotheses (3-6)).

We consider the applicative case of a sandwich piezoelectric beam of uniform width a made of a central elastic core of thickness $h_{e}$ on which two piezoelectric layers of thickness $h_{p}$ are symmetrically bonded. Analytical expressions for the beam constitutive coefficients as a function of the 3D material and geometrical properties are obtained as a function of the thickness ratio $\eta=h_{p} / h_{e}$. The two cases of in-phase and counter-phase electrical connections between the upper and lower piezoelectric layers

Table 2. Relevant material properties for the piezoelectric and elastic layers. Standard notation for piezoelectric materials is adopted. The constants $\left(s_{11}^{E}, s_{12}^{E}, d_{31}, \varepsilon_{33}^{S}\right)$ refer to the piezoelectric ceramic PZT-5H, $\left(s_{11}, s_{12}\right)$ to aluminium.

\begin{tabular}{cccccc}
\hline$s_{11}^{E}\left(m^{2} \mathrm{~N}^{-1}\right)$ & $s_{12}^{E}\left(m^{2} \mathrm{~N}^{-1}\right)$ & $d_{31}(\mathrm{~m} / \mathrm{V})$ & $\varepsilon_{33}^{T}\left(\mathrm{Fm}^{-1}\right)$ & $s_{11}\left(m^{2} \mathrm{~N}^{-1}\right)$ & $s_{12}\left(m^{2} \mathrm{~N}^{-1}\right)$ \\
\hline $16.1 \times 10^{-12}$ & $-4.67 \times 10^{-12}$ & $-320 \times 10^{-12}$ & $3.36 \times 10^{-8}$ & $14.5 \times 10^{-12}$ & $-4.78 \times 10^{-12}$ \\
\hline
\end{tabular}

are considered (extensional-electric and flexural-electric couplings, respectively). The material characteristics corresponding to aluminum and PZT-5H piezoelectric ceramic are considered (see Table 2).

\subsection{Constitutive coefficients}

For a sandwich piezoelectric beam, if a suitable global reference frame is chosen, due to the material and geometrical symmetry, the beam axis extension is mechanically uncoupled to its flexion and the beam constitutive equations (19) take the form

$$
\left[\begin{array}{c}
N_{x} \\
M_{x} \\
q
\end{array}\right]=\left[\begin{array}{ccc}
k_{N u} & 0 & e_{n V} \\
0 & k_{M w} & -e_{M V} \\
-e_{n V} & e_{M V} & \epsilon_{q V}
\end{array}\right]\left[\begin{array}{c}
u^{\prime} \\
w^{\prime \prime} \\
V
\end{array}\right]
$$

For the three models analyzed here, simple analytical expressions of the constitutive coefficients can be found. We introduce the following dimensionless parameters

$$
\tilde{\beta}_{11}=\frac{\tilde{c}_{11}}{\tilde{c}_{11}^{E}}, \tilde{\beta}_{12}=\frac{\tilde{c}_{12}}{\tilde{c}_{12}^{E}}, \tilde{\gamma}_{11}=\sqrt{\frac{\tilde{e}_{31}^{2}}{\tilde{\varepsilon}_{33}^{S} \tilde{c}_{11}^{E}}}, \tilde{\gamma}_{12}=\sqrt{\frac{\tilde{e}_{31}^{2}}{\tilde{\varepsilon}_{33}^{S} \tilde{c}_{12}^{E}}}, \tilde{\nu}=\frac{\tilde{c}_{12}}{\tilde{c}_{11}^{E}}, \hat{\beta}_{11}=\frac{\hat{c}_{11}}{\hat{c}_{11}^{E}}, \hat{\gamma}_{11}=\sqrt{\frac{\hat{e}_{31}^{2}}{\hat{\varepsilon}_{33}^{T} \hat{c}_{11}^{E}}} .
$$

Moreover the following functions are defined

$$
\omega_{\eta}(\beta)=2+\beta / \eta, \chi_{\eta}(\beta, \gamma)=2\left(1+\gamma^{2}\right)+6(1+1 / \eta)^{2}+\beta / \eta^{3} .
$$

For the model assuming null transversal strain, the following constitutive coefficients are found

$$
\begin{aligned}
& k_{N u}^{0}=\tilde{c}_{11}^{E} a h_{p} \omega_{\eta}\left(\tilde{\beta}_{11}\right), k_{M w}^{0}=\frac{c_{11}^{E} a h_{p}^{3}}{12} \chi_{\eta}\left(\tilde{\beta}_{11}, \tilde{\gamma}_{11}\right), \epsilon_{q V}^{0}=\frac{2 a}{h_{p}} \tilde{\epsilon}_{33}^{T}, \\
& e_{M V}^{0}=\omega_{\text {flex }} a \tilde{e}_{31} \frac{h_{p}+h_{b}}{2} e_{N V}^{0}=\omega_{e x t} a \tilde{e}_{31},
\end{aligned}
$$

where $\left(\omega_{\text {ext }}=2, \omega_{\text {flex }}=0\right)$ for in-phase electric connection, and $\left(\omega_{\text {ext }}=0, \omega_{\text {flex }}=2\right)$ for counterphase electric connection. The corrections to the constitutive coefficients (24) corresponding to the models assuming null transversal stress and null transversal stress resultants are reported in Table 3. 
The same functions are plotted in Figure 1 for the numerical values in Table 2 and $a=17.8 \mathrm{~mm}$, $h_{p}=0.267 \mathrm{~mm}$. Looking at Figure 1 we can observe that the mechanical and coupling constitutive coefficients estimated by the models assuming null transversal stress and null transversal stress resultants, being closed one to each other, strongly differ from those of the model assuming null transversal strain. On the other hand, sharp differences among the three models are revealed for the constitutive coefficient $\epsilon_{q V}$ which is associated to the piezoelectric capacitance. In particular models assuming either null transversal strain or null transversal stress predict a value of the piezoelectric capacitance per unit of line $\epsilon_{q V}$ which is constant with respect to $\eta$ (i.e. independent of the stiffness of the elastic substrate). For a given thickness of the piezoelectric layer, the first model gives a value of the piezoelectric capacitance which is reasonable for thick elastic substrates $(\eta \rightarrow 0)$, the second for thin ones $(\eta \rightarrow \infty)$. On the other hand, the model assuming vanishing transversal stress resultants, succeeds in providing reasonable values of the piezoelectric capacitance also for intermediate values of $\eta$ (see e.g. the plots of $\left.\epsilon_{q V}\right)$.

The equivalent piezoelectric capacitance can be measured quite easily in an experimental set-up. In order to assess the numerical results we briefly present a comparison of the measured value of $\epsilon_{q V}$ and those estimated by different models for a sandwich piezoelectric beam with a counter-phase electric connection ( $a=17.8 \mathrm{~mm}, h_{p}=0.267 \mathrm{~mm}, h_{e}=2 \mathrm{~mm}$ and material properties in Table 2). The measured value of the capacitance is $\epsilon_{q V}=2.86 \mathrm{nF} / \mathrm{mm}$. With the model assuming null transversal stress and null transversal strain one obtain, respectively, $\epsilon_{q V}=3.62 \mathrm{nF} / \mathrm{mm}(-26.9 \%)$ and $\epsilon_{q V}=$ $2.09 \mathrm{nF} / \mathrm{mm}(+26.6 \%)$. The model assuming null transversal stress resultants gives $\epsilon_{q V}=2.81 \mathrm{nF} /$ $\mathrm{mm}(-1.75 \%)$.
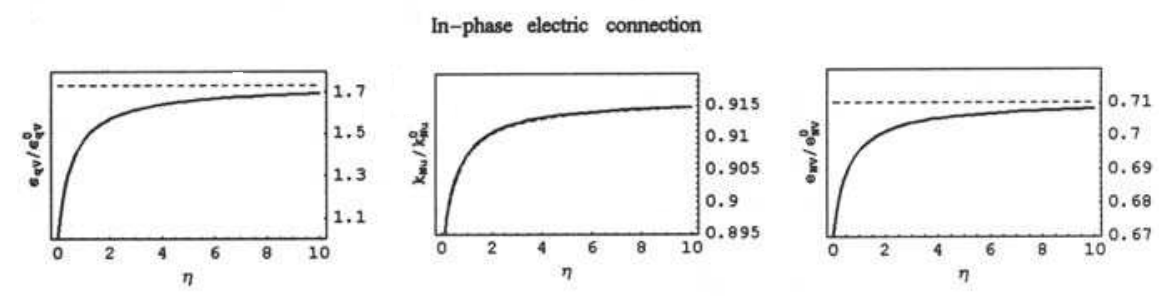

Counter-phase electric connection
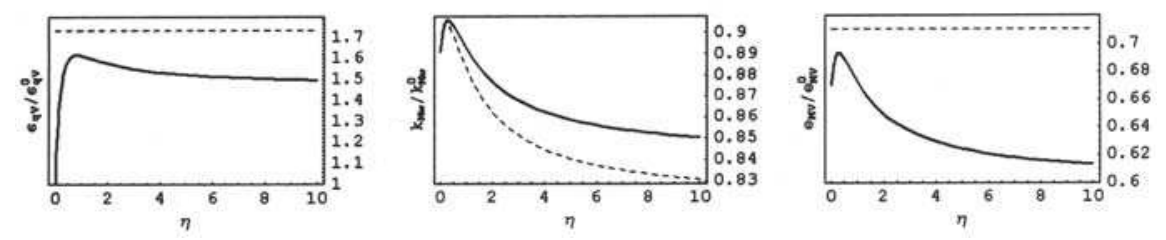

Figure 1. Sandwich beam with in-phase and counter-phase parallel electric connections. Corrections to the constitutive coefficients of the model assuming null transversal strain for model assuming null transversal stress (dashed lines) and null transversal stress resultants (continuous lines).

\subsection{Field distribution}

The through-the-thickness distribution of the 3D strain $(\overline{\mathbf{S}}, \overline{\mathbf{E}})$ and stress $(\mathbf{T}, \mathbf{D})$ taken into account at constitutive level for assigned beam generalized deformations $\left(u^{\prime}, w^{\prime \prime}, V\right)$ is presented here. Their analysis allows for understanding how the beam model mimics the three dimensional field distributions.

In order to emphasize the fundamental features of the proposed model, the through-the-thickness distribution of the transversal stress $T_{22}$ and the strain $S_{22}$ is reported in Figure 2, together with the 

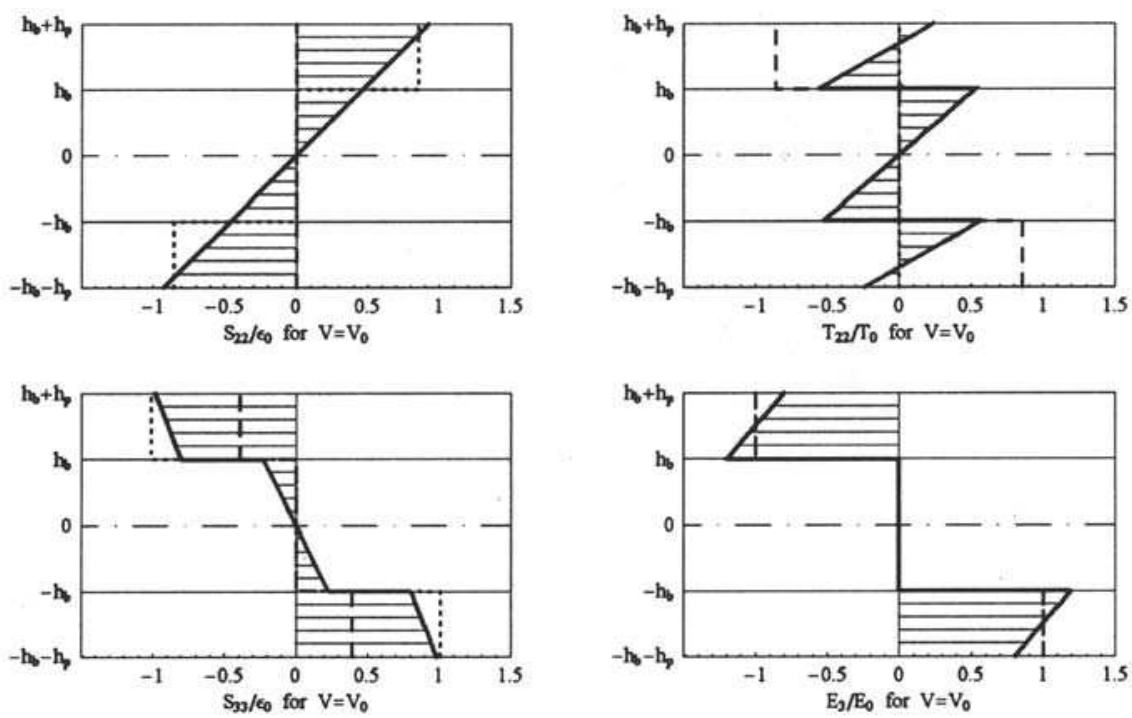

Figure 2. Through-the-thickness distribution of transversal normal stress $T_{22}$, transversal normal strain $S_{22}$, and normal strain $S_{33}$ and electric field $E_{3}$ along the thickness direction. Model assuming null transversal stress resultants (continuous line) is compared to models assuming pointwise null transversal stress (dotted line) and null transversal strain (dashed line).

Table 3. Sandwich beam with in-phase and counter-phase connections. Correction to the constitutive coefficients corresponding to null normal strain for models assuming null transversal stress (SC) and null transversal stress resultants (WC).

\begin{tabular}{|c|c|c|c|c|c|c|}
\hline & \multicolumn{3}{|c|}{ In-phase } & \multicolumn{3}{|c|}{ Counter-phase } \\
\hline & $k_{N u} / k_{N u}^{0}$ & $e_{N V} / e_{N V}^{0}$ & $\epsilon_{q V} / \epsilon_{q V}^{0}$ & $k_{M w} / k_{M w}^{0}$ & $e_{M V} / e_{M V}^{0}$ & $\epsilon_{q V} / \epsilon_{q V}^{0}$ \\
\hline sc & $\left(1-\tilde{\nu}^{2}\right) \frac{\varpi_{\eta}\left(\tilde{\beta}_{11}\right)}{\varpi_{\eta}\left(\tilde{\beta}_{11}\right)}$ & $1-\tilde{\nu}$ & $1+\tilde{\gamma}_{11}^{2}$ & $1-\tilde{\nu}^{2} \frac{\chi \eta\left(\tilde{\beta}_{11}, \tilde{\gamma}_{11}\right)}{\chi \eta\left(\tilde{\beta}_{11}, \tilde{\gamma}_{11}\right)}$ & $1-\tilde{\nu}$ & $1+\tilde{\gamma}_{11}^{2}$ \\
\hline wC & $\left(1-\tilde{\nu}^{2}\right) \frac{\varpi_{\eta}^{2}\left(\tilde{\beta}_{12}\right)}{\varpi_{\eta}^{2}\left(\tilde{\beta}_{11}\right)}$ & $1-\tilde{\nu} \frac{\varpi_{\eta}\left(\tilde{\beta}_{12}\right)}{\varpi_{\eta}\left(\tilde{\beta}_{11}\right)}$ & $1+\frac{2 \tilde{\gamma}_{11}^{2}}{\varpi_{\eta}\left(\beta_{11}\right)}$ & $1-\tilde{\nu}^{2} \frac{\chi_{\eta}^{2}\left(\tilde{\beta}_{12}, \tilde{\gamma}_{12}\right)}{\chi_{\eta}^{2}\left(\tilde{\beta}_{11}, \tilde{\gamma}_{11}\right)}$ & $1-\tilde{\nu} \frac{\chi_{\eta}\left(\tilde{\beta}_{12}, \tilde{\gamma}_{12}\right)}{\chi_{\eta}\left(\tilde{\beta}_{11}, \tilde{\gamma}_{11}\right)}$ & $1+\frac{6 \tilde{\gamma}_{11}^{2}(1+1 / \eta)^{2}}{\chi_{\eta}\left(\tilde{\beta}_{11}, \tilde{\gamma}_{11}\right)}$ \\
\hline
\end{tabular}

distribution of $S_{33}$ and $E_{3}$, normal strain and electric field along the thickness, respectively. The case of a sandwich piezoelectric beam with a counter-phase electric connection and a thickness ratio $\eta=0.5$ is considered. Comparisons with the corresponding distributions for models assuming either pointwise vanishing transversal stress or vanishing transversal strain are shown. Looking at Figure 2, we can observe that the model with weak condition on transversal stress accounts for non trivial interactions between different layers and describes: i) linear sectional deformation $S_{22}$ in the transversal direction (related to the Lagrange multipliers $\lambda$ and $\mu$ ); ii) layerwise linear transversal stress $T_{22}$; iii) linear thickness distension $S_{33}$; iv) layerwise linear distribution of the electric field $E_{3}$. On the other hand, other models neglect the transversal interactions between layers. In particular, in the model assuming null transversal stress, different layers are allowed for stretching one independently from the other ones (dotted line for the distribution of $S_{22}$ ); in model assuming null transversal strain, the transversal stress arising in the piezoelectric layers for the applied potential are not transmitted to the elastic one (dashed line for the distribution of $T_{22}$ ).

\section{CONCLUSIONS}

In this paper, models of layered piezoelectric beam accounting for two-fold electromechanical coupling were discussed. By means of a direct approach and a mixed variational principle, a model based on 
a simple Euler-Bernoulli kinematics but able to correctly mimic the through-the-thickness distribution of transversal strain and stress was presented. The main feature of the presented model is to account for transversal sectional deformations and interactions between different layers. To this end, weakened integral versions of the force balance laws in the transversal direction are enforced in the variational formulation through Lagrange multipliers method. The peculiar ability of correctly estimating, by a suitable reconstruction of the $3 \mathrm{D}$ state field distribution, the beam constitutive coefficients, in general, and the electric capacitive coefficient, in particular, was shown.

\section{References}

[1] Chopra I., "Review of state of art of smart materials structures and integrated sytems", AIAA Journal 40 (2002) 2145-2187.

[2] Saravanos D.A. and Heylinger P.R., "Mechanics and computational models for laminated piezoelectric beams, plates, and shells", Appl.Mech.Rev. 52 (1999) 305-320.

[3] Crawley E.F. and de Luis J., "Use of piezoelectric actuators as elements of intelligent structures", AIAA Journal 2510 (2002) 1373-1385.

[4] Sirohi J. and Chopra I., "Fundamental understanding of piezoelectric strain sensors", J. Intelligent Material Systems and Structures 11 (2000) 246-257.

[5] Beckert W. and Pfundtner G., "Analysis of the deformational behaviour of a bimorph configuration with piezoelectric actuation”, Smart Materials and Structures 11 (2002) 599-609.

[6] Washizu K. Variational methods in elasticity and plasticity (Pergamon Press, 1982).

[7] Teresi L. and Tiero A., "On variational approaches to plate models”, Meccanica 32 143-156.

[8] Lembo M.and Podio-Guidugli P., "Internal Constraints, reactive stresses and the Timoshenko beam theory", Journal of Elasticity 65 (2001) 131-148.

[9] Ikeda T. Fundamentals of piezoelectricity (Oxford University press, Oxford, 1990). 